\title{
Clear Demonstration of Different Mechanisms of Severe Mitral Regurgitation Caused by the Mitral Ring Dehissence during Transesophageal Echocardiography
}

\author{
Mustafa Ferhat Keten ${ }^{1}$, Berhan Keskin ${ }^{2}$, Ahmet Karaduman ${ }^{1}$, İsmail Balaban ${ }^{1}$, and \\ Gokhan Kahveci ${ }^{3}$ \\ ${ }^{1}$ Kartal Kosuyolu Training and Research Hospital \\ ${ }^{2}$ Kartal Kosuyolu Yuksek Ihtisas Egitim ve Arastirma Hastanesi \\ ${ }^{3}$ Rize State Hospital
}

June 28, 2020

\begin{abstract}
Mitral ring dehiscence is a rare clinical condition and may result in the recurrence of severe mitral regurgitation(MR). Echocardiography has a critical role in the diagnosis. In this case, a patient with prosthetic mitral ring dehiscence was evaluated with transesophageal echocardiography and demonstrative images have been obtained. Interestingly, two different MR jets, first one from the perimitral ring and the second one from the basal portion of the posterior mitral leaflet(PML) were present. Most likely, suturing of the mitral ring to the basal portion of the PML, which is an unexpected condition, caused to MR after the ring dehiscence
\end{abstract}

\section{Case}

A 55-year-old male patient was admitted to the outpatient clinic with intolerable dyspnea which occurs with minimal effort. He had a mitral repair with a Memo annuloplasty ring due to a severe mitral regurgitation (MR) associated with annular dilation caused by atrial fibrillation 3 years ago and there was no residual MR in the postoperative echocardiography. Transthoracic echocardiography revealed normal left ventricular ejection fraction (\%55) and moderate-severe MR. Transesophageal echocardiography (TEE) was decided as a next step to clarify the mechanism of the mitral regurgitation (MR). Rocking prosthetic ring and dehiscence were present in the mid-esophageal two- and three-dimensional TEE views respectively with a severe mitral regurgitation (figure 1a, video 1,2). There were two mitral regurgitation jets. It was observed that in the mid-esophageal TEE views, the first jet was originating from the perimitral ring and the second jet was arising from the basal portion of the posterior mitral leaflet which was suitable with the location of the mitral ring suture(figure $1 \mathrm{~b}$, video 3 ). Interestingly, dehiscence of the mitral ring possibly caused the occurrence of a defect in the posterior mitral leaflet in the junction zone and this defect emerged as another source of MR(figure 1c-1d, video 4). Surgical treatment was planned for the patient due to the presence of NYHA class III symptoms and severe MR.

Ring annuloplasty is mainly used to fix MR caused by the geometrical changes in the left ventricle which may result in annular dilation. Ring dehiscence is a rare clinical entity that usually leads to severe MR and requires urgent or emergent surgical reoperation(1). Endocarditis, trauma, or procedure-related issues may be responsible to trigger the dehiscence process and progressive left ventricular geometric remodeling may also provoke recurrence $(2,3)$. Characteristics of the tissue at the ring attachment areas are another important factor so, weak and calcified tissues are more prone to separation from the ring(3). Furthermore, attachment 
of the mitral ring to the basal portion of the PML in the previous surgery may cause a predisposition to ring dehiscence, and the suture site on the PML behaved as an independent source of MR besides the regurgitation jet from the perimitral ring. In this case, two- and three-dimensional echocardiographic demonstrative images are presented to highlight this rare clinical condition.

\section{References}

1. Noack T, Kiefer P, Vivell N, et al. Annuloplasty ring dehiscence after mitral valve repair: incidence, localization and reoperation. Eur J Cardiothorac Surg . 2020;57(2):300-307.

2. Mashicharan M, Loke I, Zlocha V et al. Separation anxiety: Extreme dehiscence of a mitral annuloplasty ring. Echocardiography . 2019;36(6):1219-1221.

3. Aggarwal G, Schlosshan D, Mathur G et al. Recurrent ischaemic mitral regurgitation post mitral annuloplasty due to suture dehiscence evaluated using real time three dimensional transoesophageal echocardiography. Heart Lung Circ . 2012;21(12):844-846.

\section{Figure Legends}

Figure 1a: Three-dimensional transoesophageal echocardiography (TEE) imaging of the mitral ring dehiscence.

Figure 1b: The first jet was originating from the perimitral ring(arrow) and the second jet(asterisk) was arising from the basal portion of the posterior mitral leaflet.

Figure 1c: Image of the defect in the basal portion of the posterior mitral leaflet in the mid-esophageal TEE view.

Figure 1d: Rise of the mitral regurgitation jet from the defect in the basal portion of the posterior mitral leaflet possibly occurred after the separation of mitral ring suture.

Video 1: Rocking motion of the prosthetic mitral ring was present in the bicommissural TEE view.

Video 2: Three-dimensional transesophageal echocardiography (TEE) imaging of the mitral ring dehiscence.

Video-3: Severe mitral regurgitation(MR) was observed in the mid-esophageal TEE views with two distinct jets, the first MR jet was originating from the perimitral ring(asterisk) and the second one was arising from the basal portion of the posterior mitral leaflet(arrow) which was suitable with the location of the mitral ring suture.

Video-4: Dehiscence of the mitral ring possibly caused the occurrence of a defect in the posterior mitral leaflet in the junction zone and this defect emerged as another source of MR. 


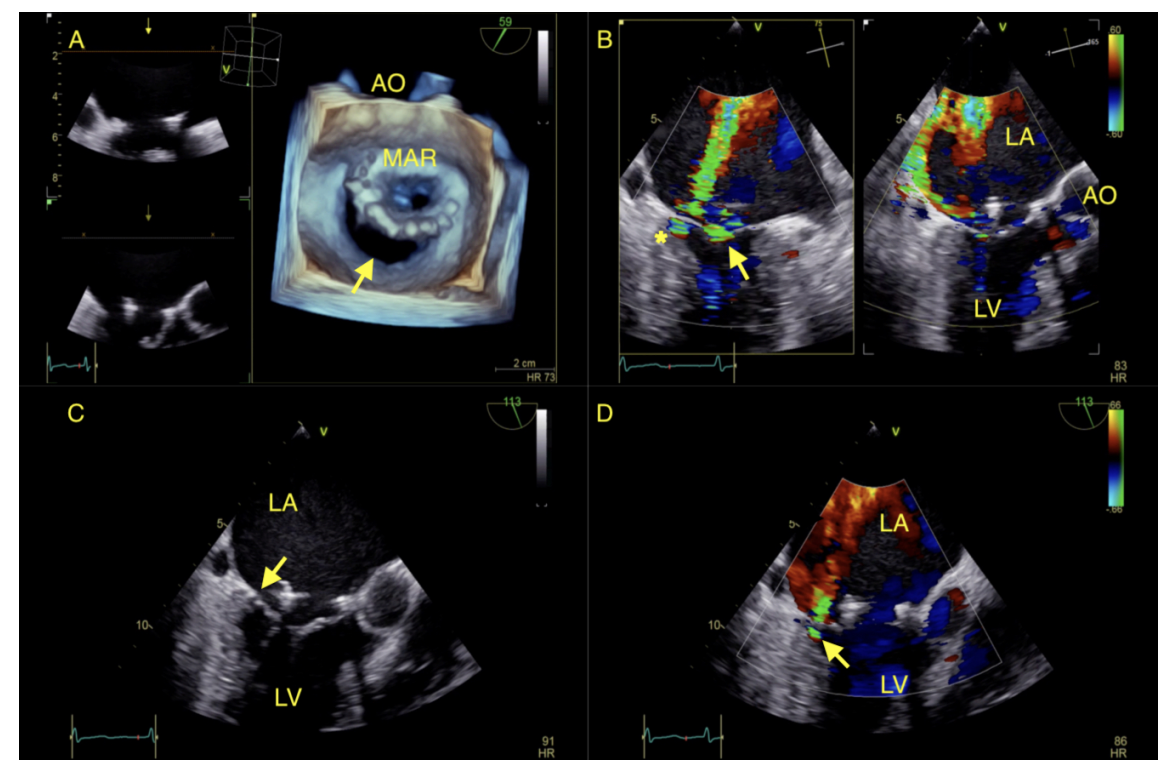

